\title{
ANÁLISIS DE LA SERIE TEMPORAL DE LA ESTACIÓN DE MONITOREO CONTINUO CUEC - REGME
}

\author{
ANALYSIS OF TIME SERIES OF THE CONTINUOUS MONITORING STATION \\ CUEC-REGME
}
MARCO P. LUNA ${ }^{1}$, ALEJANDRA STALLER ${ }^{2}$, KERBY HARNISTH ${ }^{1,}$ DARÍO ECHEVERRÍA $^{1}$, ROGER CASTRO ${ }^{1}$, ÓSCAR PORTILLA ${ }^{1}$

${ }^{1}$ UNIVERSIDAD DE LAS FUERZAS ARMADAS ESPE, Av. General Rumiñahui s/n y Ambato, P.Box 171-5-231B, Sangolquí, Ecuador.

${ }^{2}$ UNIVERSIDAD POLITÉCNICA DE MADRID, ETSI Topografía, Geodesia y Cartografía. Campus Sur - UPM, Madrid, Spain

Recibido: 29 de septiembre de 2018 / Aceptado: 23 de diciembre de 2018

\begin{abstract}
RESUMEN
En el presente estudio se realiza un análisis exhaustivo de la serie temporal de la estación de monitoreo contínuo CUEC en el periodo 2008-2014. Para tal efecto se obtuvieron soluciones diarias de coordenadas con sus respectivas precisiones en cada una de sus componentes tanto horizontales como verticales, para posteriormente realizar un análisis riguroso de la serie mediante una descomposición aditiva determinando la estacionalidad o periodicidad, la tendencia y el tipo de ruido presente en la serie. Previo a la descomposición se realizó una limpieza o depuración de los datos mediante la detección y corrección de saltos en la serie y eliminación de valores atípicos. Para la determinación de la tendencia utilizamos una regresión lineal ponderada cuya solución se obtuvo mediante un ajuste mínimo cuadrático, en la determinación de la estacionalidad se realizó un análisis espectral de la serie en el dominio de las frecuencias, ya que esta es una medidad que sirve para representar los ciclos, y en cuanto a la obtención del ruido se lo realizó mediante el estudio del espectro de potencia. Los resultados obtenidos muestran que la estación CUEC tiene una periodicidad anual (1.03 años) en todas sus componentes y un tipo de ruido gaussiano fraccionario con un índice espectral entre 0 y -1 , considerando que estos valores no reflejan el verdadero tipo de ruido existente, debido principalmente a un alto porcentaje de datos perdidos.
\end{abstract}

Palabras clave: análisis espectral, tendencia, estacionalidad, ruido

\begin{abstract}
In the present study an exhaustive analysis of the time series of the continuous monitoring station CUEC in the period 2008-2014 is carried out. For this purpose, daily solutions of coordinates were obtained with their respective precisions in each one of their components, both horizontal and vertical, to subsequently carry out a rigorous analysis of the series by means of an additive decomposition determining the seasonality or periodicity, the tendency and the type of noise present in the series. Prior to decomposition, data was cleaned or purified by detecting and correcting jumps in the series and eliminating outliers. For the determination of the trend we used a weighted linear regression whose solution was obtained by a minimum square adjustment, in the determination of the seasonality a spectral analysis of the series was made in the frequency domain, since this is a measure that serves to represent the cycles, and in terms of obtaining the noise was made by studying the power spectrum. The results obtained show that the CUEC station has an annual periodicity (1.03 years) in all its components and a type of fractional Gaussian noise with a spectral index between 0 and -1 , considering that these values do not reflect the true existing noise type, mainly due to a high percentage of lost data.
\end{abstract}

Key words: spectral analysis, trend, seasonality, noise 


\section{INTRODUCCIÓN}

El objetivo principal del análisis de series temporales es frecuentemente encontrar un modelo óptimo que describa los procesos estocásticos que se pueden haber generado en las series. Lo que usualmente se hace es separar la información de la serie de tiempo en una parte determinística llamada "señal" y otra parte estocástica conocida como "ruido" (Peña, 2010).

La serie obtenidas en este estudio corresponden a datos diarios de la estación de monitoreo contínuo CUEC, perteneciente a la Red GNSS de Monitoreo Continuo del Ecuador (REGME), tomados en el periodo 29-10-2008 al 31-12-2014, semanas GPS 1503 hasta 1825. Los resultados se obtuvieron en coordenadas cartesianas $(X, Y, Z)$ en el sistema ITRF2008 (IGb08). Para un mejor análisis e interpretación de las series temporales, las coordenadas cartesianas geocéntricas globales $(X, Y, Z)$ obtenidas como solución final en el procesado con Bernese, se convierten a coordenadas locales, respecto de un sistema de coordenadas topocéntrico, centrado en un punto de la superficie terrestre y orientado según la Figura 1. Este tipo de coordenadas nos proporciona una idea más clara del comportamiento de cada serie en sus respectivas componentes. Otra ventaja importante es el hecho de trabajar con valores pequeños, ya que estas pueden ser centradas en cero, lo que facilita su estudio.

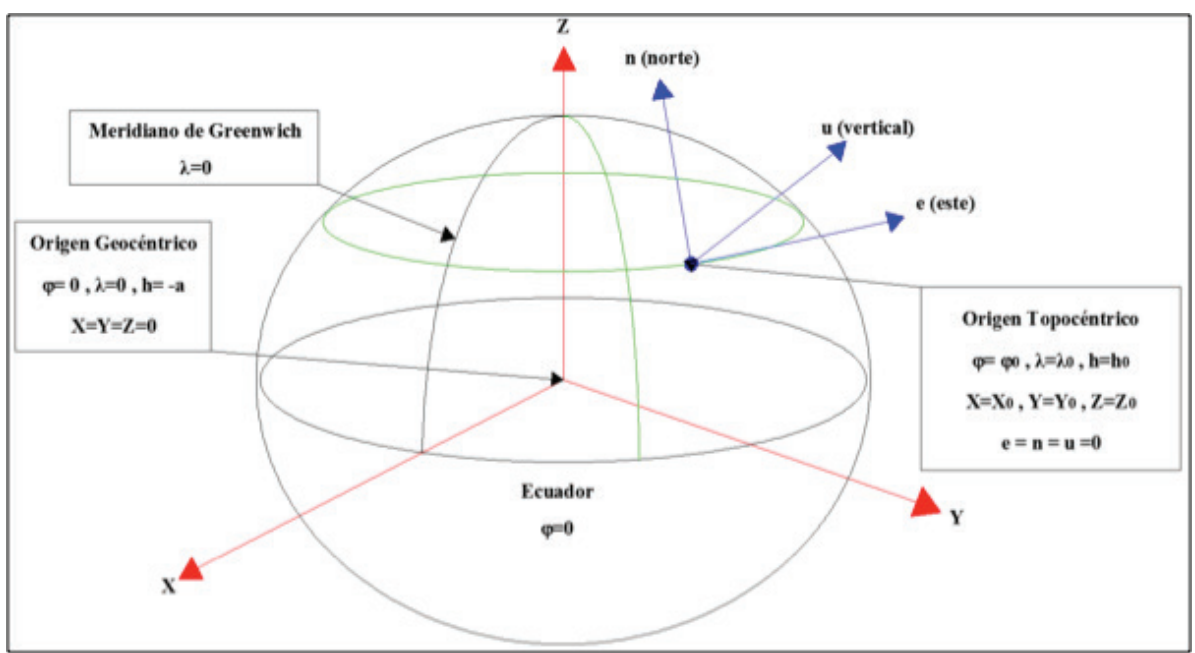

Figura 1. Sistema de coordenadas cartesianas globales $(X, Y, Z)$ y topocéntricas locales (e, n, u). Modificada de Drewes (2014).

Las coordenadas de la serie temporal en este trabajo, cambia con el tiempo principalmente por el movimiento de las placas tectónicas. Existen además errores en su determinación debido a diferentes causas de tipo físico como instrumentales que en ciertos casos hace que se tengan valores anómalos respecto a los de su alrededor. Así pues, antes de realizar propiamente el análisis de la serie, es necesario trabajar con una serie depurada o limpia, es decir que estén libres de valores atípicos (outliers) y de saltos (offsets). 
La estrategia utilizada es la descomposición aditiva de la serie que considera que los datos se generan como la suma de tres factores que son: la tendencia, la estacionalidad o periodicidad y el ruido. Los métodos clásicos de descomposición suponen que tanto la tendencia como la estacionalidad son deterministas y el ruido la parte aleatoria o estocástica (García, 2016).

En el presente estudio, el análisis y la determinación del tipo de ruido se realiza mediante el análisis del espectro de potencia aproximado por una ley de potencia dependiente de la frecuencia, propuesto por Agnew (1992). Además, se calculan la estacionalidad y la tendencia de cada serie. La periodicidad o estacionalidad se determina mediante el análisis espectral para series temporales con datos que no están igualmente espaciados, según el procedimiento propuesto por Lomb (1976) y la tendencia de la serie se determina utilizando regresión lineal ponderada.

\section{MÉTODOS}

\section{ANÁLISIS DE SERIES TEMPORALES}

Detección y Corrección De Outliers Y Offsets

En las series de tiempo de datos de estaciones de monitoreo continuo suelen observarse dos tipos de valores anómalos: los saltos en las series (offsets) y los valores atípicos (outliers). La secuencia en la que tratamos estos valores es en primer lugar detectar y corregir los offsets, si los hubiese, para posteriormente eliminar los valores atípicos encontrados.

El método utilizado para la corrección del offset se basa en calcular las tendencias de las series antes y después del offset mediante un ajuste de mínimos cuadrados, obteniéndose las ecuaciones de las rectas para cada lado del offset.(García, 2016).

El valor de salto del offset corresponde a las diferencias entre los valores obtenidos al evaluarse las ecuaciones de las rectas en el tiempo donde inició el offset; así tenemos:

$$
\Delta\left(\mathrm{t}_{\text {offs }}\right)=\mathrm{y}_{1}\left(\mathrm{t}_{\text {offs }}\right)-\mathrm{y}_{2}\left(\mathrm{t}_{\text {offs }}\right)
$$

En cuanto a los outliers o valores atípicos en las series temporales pueden existir dos tipos: aquellos en los que el dato es anómalo respecto a los de su alrededor, o también puede darse el caso en que el valor de la coordenada no sea discordante con el resto, pero el error con respecto a la tendencia de la serie sí que lo sea y en este caso también se puede considerar un valor atípico. Por tanto, para la determinación de los mismos se va a proceder a su detección utilizando para cada caso técnicas diferentes.

Es evidente que nuestras series no son estables en el tiempo debido a que las coordenadas de la estación se encuentran sobre la superficie de la corteza en placas tectónicas que están en continuo movimiento. Éstas, en particular, tienen una tendencia creciente o decreciente, 
obteniéndose series no estacionarias en la media. Así pues, para no involucrar a la tendencia desde un inicio en la eliminación de los valores atípicos, recurrimos a la utilización de los procesos integrados, que para este estudio resultó ser de grado 1, donde el orden de integración es el número de diferencias necesarias para obtener un proceso estacionario.

Es así que, para una serie temporal de un conjunto de observaciones, $Z_{\mathrm{i}}=Z_{1}, Z_{-} 2, \ldots Z_{\mathrm{N}}$ en los instantes de tiempo $t_{i}$ con $i=1,2,3, \ldots \mathrm{N}$ siendo el tamaño muestral de la serie, el proceso integrado de orden 1 está dado por:

$$
\Delta Z_{i}=Z_{1}-Z_{2}, Z_{2}-Z_{3}, Z_{3}-Z_{4}, \ldots, Z_{N-1}-Z_{N}
$$

La nueva serie oscila alrededor de una media constante, considerándose valores atípicos aquello que sobrepasan el siguiente intervalo:

$$
\overline{\Delta Z_{\iota}} \pm 3 \cdot \sigma_{\Delta z}
$$

siendo $\overline{\Delta Z_{\imath}}$ y $\sigma_{\Delta z}$ la media y desviación estándar de los procesos integrados.

Una vez depurada la serie usando los procesos integrados de orden 1 , se determina la tendencia de la serie mediante regresión lineal simple y se consideran valores atípicos aquellos residuales que sobrepasen tres desviaciones estándar de los errores (Mao et al., 1999).

Descomposición aditiva de las series temporales

Es ampliamente conocido que cualquier señal periódica está compuesta por la frecuencia principal, así como sus armónicos más altos. Debido a esto, asumimos que la serie de tiempo contiene tanto la parte determinista mediante el modelo funcional que incluye la tendencia y la estacionalidad, así como el ruido de fondo (Amiri-Simkooei et al., 2007). Una vez eliminados y corregidos los valores atípicos y saltos se obtiene una serie depurada o limpia. El propósito fundamental de este estudio es obtener un modelo de la serie que mejor se ajuste a ella, tomando en consideración la tendencia, variaciones estacionales y el tipo de ruido, de tal forma que la suma de todas estas componentes represente la serie temporal real. El modelo teórico es

$$
\mathrm{y}_{\mathrm{t}}=\mathrm{T}_{\mathrm{t}}+\mathrm{S}_{\mathrm{t}}+\mathrm{N}_{\mathrm{t}}
$$

donde

$\mathrm{y}_{\mathrm{t}}=$ Valor observado

$\mathrm{T}_{\mathrm{t}}=$ Tendencia

$\mathrm{S}_{\mathrm{t}}=$ Estacionalidad

$\mathrm{N}_{\mathrm{t}}=$ Ruido, componente irregular, fluctuaciones aleatorias no explicadas por otros componentes. 
Al introducir la estacionalidad y el tipo de ruido tendremos una mejor estimación del parámetro que querramos estudiar, como por ejemplo la velocidad y su precisión. A continuación, se describe el proceso llevado a cabo para obtener el modelo propuesto.

\section{Tendencia}

La tendencia de una serie viene dada por el movimiento a largo plazo de los datos que la conforman, caracterizada por un patrón gradual y variaciones de la propia serie, que en nuestro caso se consideran como consecuencia del movimiento y deformación de la corteza terrestre que afectan al crecimiento o disminución de la misma.

Por lo general, la tendencia a largo plazo se aproxima a una línea recta que describe el movimiento de una determinada estación. El modelo funcional será:

$$
\mathrm{T}(\mathrm{t})=\mathrm{y}_{\mathrm{o}}+\mathrm{rt}+\varepsilon
$$

donde $\mathrm{y}_{\mathrm{o}}$ representa la ordenada en el origen, $r$ la pendiente, $t$ el instante de tiempo y $\varepsilon$ el error de ajuste. Nuestros datos corresponden a las posiciones que tienen asociadas una precisión procedente de la matriz varianza-covarianza del procesado de los datos. Utilizamos esta precisión para obtener, mediante una regresión lineal ponderada, la tendencia de la serie donde los pesos están dados por:

$$
W_{i}=1 / \sigma_{i}
$$

La solución se determina utilizando un ajuste mínimo cuadrático mediante el siguiente sistema de matrices:

$$
X=\left(A^{\prime} W A\right)^{-1} A^{\prime} W Y
$$

siendo $X$ la matriz de los parámetros a determinar $\left(\mathrm{y}_{\mathrm{o}}, \mathrm{r}\right), A$ la matriz de los coeficientes, $W$ matriz diagonal de pesos e $Y$ la matriz de valores observados (Wolf y Ghilani, 1997).

\section{Estacionalidad}

La estacionalidad de una serie se refiere a las fluctuaciones periódicas u oscilaciones que se repiten cada cierto periodo de tiempo. La componente estacional de una serie debe recoger estas oscilaciones y deben incorporarse al modelo de las series temporales. La importancia de modelar esta señal estacional radica en que puede afectar a los parámetros de interés estimados a partir de las series de tiempo, en particular la velocidad de la estación (Blewitt and Lavallée, 2002; Bos et al., 2008). Para obtener la estacionalidad es necesario realizar el análisis espectral de las series de tiempo en el dominio de las frecuencias, ya que esta es una medida que sirve para representar ciclos. 
El promedio estadístico de una determinada señal o tipo de señal (incluido el ruido), tal como se analiza en términos de su contenido de frecuencia, se denomina espectro. El espectro de potencia de una serie temporal describe la distribución de potencias en componentes de frecuencia que componen esa señal. Se pueden usar varios términos para referirse al espectro de potencia, uno de los más utilizados es la densidad espectral de potencia (PSD, Power Spectral Density). Para muestras discretas la PSD se generaliza a series de tiempo finitas como señal muestreada a intervalos discretos de tiempo en un periodo de medición neto.

Cuando se tiene el espectro de potencia, se pueden calcular varias características útiles de la señal de entrada, tales como potencia, frecuencia, armónicos y nivel de ruido. El cálculo del espectro de potencia se realiza con los residuales de las series, es decir con la serie eliminada la tendencia. En el apartado anterior se vio cómo determinar los valores de la pendiente $r$ y la ordenada en el origen mediante el análisis de tendencia, por tanto el valor de los residuales sería:

$$
v_{i}=y_{\text {iobs }}-\left(y_{o}+r t_{i}\right)
$$

Para determinar el espectro de potencia por medio de un periodograma para series discretas igualmente espaciadas se define la Transformada de Fourier como (Scargle, 1982):

$$
P\left(f_{n}\right)=\frac{1}{N}\left[\left(\sum_{i=1}^{N} v_{i} \cos \left(2 \pi i f_{n}\right)\right)^{2}+\left(\sum_{i=1}^{N} v_{i} \sin \left(2 \pi i f_{n}\right)\right)^{2}\right]
$$

donde $f_{n}=n / T$, T es el periodo fundamental, $v_{i}$ es el residuo y $n=1,2, \ldots N / 2$.

Fourier demostró que toda función periódica puede representarse como sumas de funciones sinusoidales de distinta amplitud y frecuencia. Este resultado sugiere generalizar el análisis realizado para un ciclo permitiendo que la función periódica sea la suma de varias funciones armónicas con distintas frecuencias o periodos, por tanto, el modelado de la estacionalidad estará representado por una función armónica de periodo $T$. Así pues, la alternativa más simple para representar la estacionalidad como una función periódica es suponer una función armónica, como el seno y el coseno.

Este procedimiento es ampliamente utilizado cuando las series tienen datos igualmente espaciados (Mao et al., 1999). Sin embargo, nuestra serie corresponde a observaciones influenciadas por varios factores que producen datos anómalos los cuales deben ser 
eliminados previo a su análisis para que no influyan en la determinación de los parámetros a estimar. Además, hay vacíos o gaps en las series debido a múltiples factores como pérdida de datos o problemas en las estaciones que hacen que no tengamos información para todos los días procesados y consecuentemente no estén igualmente espaciados. Por tanto, para obtener el espectro de potencia por medio del periodograma para este caso Mao et al. (1999) recomiendan seguir el procedimiento propuesto por Lomb (1976) para series que no están igualmente espaciadas, donde se calcula mediante la inclusión del retardo definido por:

$$
\tan (4 \pi f \tau)=\sum_{i=1}^{N} \sin \left(4 \pi t_{i} f\right) / \sum_{i=1}^{N} \cos \left(4 \pi t_{i} f\right)
$$

De esta ecuación se despeja el valor de $\tau$ y se reemplaza en la ecuación (11) para obtener los valores del espectro de potencia (Press et al., 2007).

$$
P(f)=\frac{1}{2}\left\{\frac{\left[\sum_{i=1}^{N} v_{i} \cos \left(2 \pi f\left(t_{i}-\tau\right)\right)\right]^{2}}{\sum_{i=1}^{N} \cos ^{2}\left(2 \pi f\left(t_{i}-\tau\right)\right)}+\frac{\left[\sum_{i=1}^{N} v_{i} \sin \left(2 \pi f\left(t_{i}-\tau\right)\right)\right]^{2}}{\sum_{i=1}^{N} \sin ^{2}\left(2 \pi f\left(t_{i}-\tau\right)\right)}\right\}
$$

Los valores más altos del espectro de potencia representan las frecuencias y periodos predominantes, obteniendo de esta manera el periodograma de la serie en estudio.

Las variaciones estacionales están dadas por:

$$
\begin{gathered}
S(t)=\sum_{k=1}^{p}\left(A_{k} \cdot \sin \left(2 \pi f_{k} t\right)+B_{k} \cdot \cos \left(2 \pi f_{k} t\right)\right)+\varepsilon \\
A=\frac{2}{N} \sum_{i=1}^{N} v_{i} * \sin \left(2 \pi f t_{i}\right) \quad B=\frac{2}{N} \sum_{i=1}^{N} v_{i} * \cos \left(2 \pi f t_{i}\right)
\end{gathered}
$$

Para el análisis de nuestra serie hemos tomado hasta tres armónicos.

Ruido - Análisis espectral

La comprensión del contenido del ruido es muy importante para que se puedan asignar incertidumbres realistas a los parámetros estimados en las series de tiempo. Existen varios factores que hacen difícil determinar la fuente que los genera y las incertidumbres en las observaciones de las estaciones de monitoreo continuo. 
En los últimos años se ha detectado y estudiado la correlación temporal de las coordenadas, ya que su determinación se ve afectada por numerosos efectos geofísicos y/o meteorológicos que dependen del tiempo (García, 2016). Ya algunos autores han determinado la presencia de ruido correlado con el tiempo y su efecto en la estimación de la incertidumbre en las series con observaciones GPS. Varios autores indican que éstas son subestimadas si solo se considera el ruido blanco en las series (i.e. Johnson y Agnew, 1995; Zhang et al., 1997; Mao et al., 1999; Williams et al., 2004; Hackl et al., 2011).

El análisis espectral de una serie cronológica es el método tradicional utilizado para determinar las características de las observaciones, entre ellas la de establecer el tipo de ruido existente en cada serie mediante el estudio del espectro de potencia. El espectro de potencia $P$, de muchos fenómenos geofísicos están bien aproximado por una ley de potencia dependiente de la frecuencia de la forma (Agnew, 1992):

$$
P(f)=P_{o} f / f_{o}^{\alpha}
$$

donde $f$ es la frecuencia temporal, $\mathrm{P}_{0} \mathrm{y} f_{o}$ son constantes de normalización y $\alpha$ es el índice espectral. Por lo general el índice espectral no es un número entero y sus valores varían entre $0<\alpha<-3$, que es el que aparece en la mayoría de los fenómenos geofísicos (Agnew, 1992). El conocimiento del índice espectral es importante porque permite identificar el tipo de ruido presente en la serie y así poder modelarlo y tenerlo en cuenta al momento de estimar las velocidades y mejorar su precisión.

El método para la obtención del valor de es ajustando una recta $\alpha \cdot \log (\mathrm{P}(\mathrm{f}))-\log$ (f), siendo la pendiente el valor correspondiente al índice espectral. Si $-1<\alpha<0$ recibe el nombre de ruido gaussiano fraccionario y se suele considerar que es estacionario, es decir, sus propiedades estadísticas son invariantes con el tiempo.

Existen tres tipos de ruidos específicos que se encuentran dentro del estudio de series temporales en observaciones de estaciones de monitoreo continuo, estos son: ruido blanco, ruido de parpadeo o rosa (flicker) y ruido rojo, browniano o de camino aleatorio (random walk) cuando el índice espectral toma valores de $0,-1$ y -2, respectivamente.

\section{RESULTADOS Y DISCUSIÓN}

\section{OFFSETS Y OUTLIERS}

Una forma clara de identificar los offsets es mediante la determinación visual en el gráfico de las series temporales, para posteriormente recurrir a los archivos y obtener la fecha exacta del salto. La Figura 2 muestra la serie temporal de la estación CUEC afectada por un offset con su correspondiente corrección en todas sus componentes. 


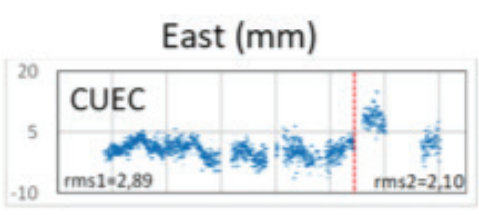

20082009201020112012201320142015

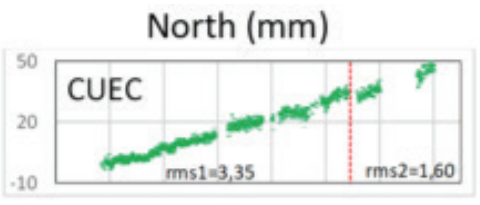

20082009201020112012201320142015

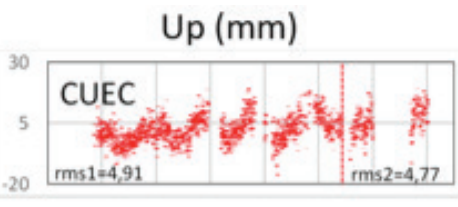

20082009201020112012201320142015

Figura 2. Estación CUEC con offset y outliers. La línea roja representa el momento en que se produjo el offset, se presenta el error cuadrático medio antes de la eliminación de outliers y corrección de offset (rms1) y el error cuadrático medio de los datos depurados (rms2).

La Tabla 1 muestra el valor de offsets para cada una de sus componentes, siendo mayor en la componente este con un valor de $10.07 \mathrm{~mm}$, seguido por la componente vertical con $-7.19 \mathrm{~mm}$ y un valor menor para la componente norte con $-3.52 \mathrm{~mm}$.

Tabla 1. Fecha y valor de los offsets detectados en la estación CUEC.

\begin{tabular}{|c|c|c|c|c|c|}
\hline \multirow{2}{*}{ ESTACIÓN } & \multicolumn{2}{|c|}{ FECHAS } & \multicolumn{3}{|c|}{ VALORES DE OFF- } \\
\cline { 2 - 6 } & $\begin{array}{c}\text { Primer } \\
\text { Offset }\end{array}$ & $\begin{array}{c}\text { Segundo } \\
\text { offset }\end{array}$ & $\begin{array}{c}e \\
(\mathrm{~mm})\end{array}$ & $\begin{array}{c}n \\
(\mathrm{~mm})\end{array}$ & $\begin{array}{c}u \\
(\mathrm{~mm})\end{array}$ \\
\hline \multirow{2}{*}{ CUEC } & 04 -jun-13 & $\begin{array}{c}28 \text {-dic- } \\
13\end{array}$ & $\mathbf{1 0 . 0 7}$ & -3.52 & -7.19 \\
\hline
\end{tabular}

En cuanto a los outliers o valores atípicos, como resultado del algoritmo de limpieza utilizado se eliminó un promedio de un 5.8\% de datos (Tabla 2). Estos valores no están acordes con el porcentaje obtenido en otros estudios, como por ejemplo Nikolaidis (2002), quien alcanzó el nivel promedio de limpieza de datos de 1-4\%. El método empleado por este autor se basa en la utilización de la mediana y el rango intercuartílico (IQR) como estadísticos para determinar los valores atípicos.

Tabla 2. Porcentaje de valores atípicos eliminados para cada estación.

\begin{tabular}{cccc}
\hline ESTACIÓN & $\begin{array}{c}\text { \# datos } \\
\text { originales }\end{array}$ & $\begin{array}{c}\text { \# datos de- } \\
\text { purados }\end{array}$ & $\begin{array}{c}\text { \% datos elimi- } \\
\text { nados }\end{array}$ \\
\hline CUEC & 1547 & 1458 & 5.8 \\
\hline
\end{tabular}

Los valores atípicos de las series de la estación CUEC posiblemente indican un cambio temporal, como un movimiento repentino del monumento geodésico, que pronto se recuperaría. 


\section{TENDENCIA}

La tendencia de la serie con sus incertidumbres es una primera aproximación de la velocidad. La Tabla 3 muestra la estimación de la velocidad de la estación CUEC con sus respectivas precisiones. Los valores del error medio cuadrático $(r m s)$ y el error cuadrático medio ponderado (wmrs), llamado repetibilidad, son valores de precisiones más realistas. Las diferencias encontradas entre estos dos parámetros no son significativas por ser los pesos semejantes entre sí. Los valores de wrms corresponden a la repetibilidad a largo plazo de la serie y está en el orden de $1.94 \mathrm{~mm}$ y $1.63 \mathrm{~mm}$ para la componente horizontal $(e, n)$ y entre $4.80 \mathrm{~mm}$ para la componente vertical $(u)$, siendo estos resultados más conservadores.

En cuanto a las incertidumbres asociadas a la estimación de las velocidades para la componente horizontal son de $0.06 \mathrm{~mm} /$ año y $0.05 \mathrm{~mm} / \mathrm{año}$, y para la componente vertical de $0.15 \mathrm{~mm} /$ año.

Tabla 3. Primera aproximación de las velocidades GNSS de las estaciones REGME. Las componentes de velocidad este, norte y vertical ( $\mathrm{Ve}, \mathrm{Vn}, \mathrm{Vu}$ ) y las incertidumbres al $95 \%$ de

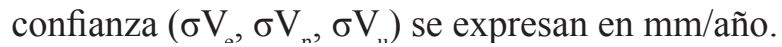

\begin{tabular}{|c|c|c|c|c|c|c|c|c|c|c|c|c|c|c|c|}
\hline \multirow[b]{2}{*}{ Estación } & \multicolumn{3}{|c|}{ Posición } & \multirow{2}{*}{$\begin{array}{c}\mathrm{Ve} \\
(\mathrm{mm} / \mathrm{a})\end{array}$} & \multirow{2}{*}{$\begin{array}{c}\mathrm{Vn} \\
(\mathrm{mm} / \mathrm{a})\end{array}$} & \multirow{2}{*}{$\begin{array}{c}\mathrm{Vu} \\
(\mathrm{mm} / \mathrm{a})\end{array}$} & \multirow[b]{2}{*}{$\sigma \mathrm{Ve}$} & \multirow[b]{2}{*}{$\sigma \mathrm{Vn}$} & \multirow[b]{2}{*}{$\sigma \mathrm{Vu}$} & \multirow[b]{2}{*}{$\mathrm{rms}_{\mathrm{e}}$} & \multirow[b]{2}{*}{$\mathrm{rms}_{\mathrm{n}}$} & \multirow[b]{2}{*}{$\mathrm{rms}_{\mathrm{u}}$} & \multirow[b]{2}{*}{ wrms, } & \multirow[b]{2}{*}{$\mathrm{wrms}_{\mathrm{n}}$} & \multirow[b]{2}{*}{ wrms $_{u}$} \\
\hline & $\begin{array}{l}\text { Long. } \\
\left({ }^{\circ}\right)\end{array}$ & $\begin{array}{l}\text { Lat. } \\
\left({ }^{\circ}\right)\end{array}$ & $\begin{array}{l}\text { Elev. } \\
\text { (m) }\end{array}$ & & & & & & & & & & & & \\
\hline CUEC & -79.00 & 2.883 & 2631.2 & -0.30 & 7.89 & 1.90 & 0.06 & 0.05 & 0.15 & 1.97 & 1.68 & 4.88 & 1.94 & 1.63 & 4.80 \\
\hline
\end{tabular}

Los valores del error medio cuadrático y la repetibilidad a largo plazo corresponden al error de ajuste respecto a la recta de regresión, estas precisiones están más acordes con la repetibilidad semanal o repetibilidad a corto plazo, como lo indica la Tabla 3, pues es conocido que los valores de la repetibilidad son de 2 a 20 veces peores que la desviación estándar para la mayoría de las estaciones, siendo mayor para la componente vertical, que en nuestro caso es de aproximadamente 10 veces. En cambio, los valores de la precisión de las coordenadas están más acordes a la estimación de la incertidumbre de la tendencia. En la Tabla 3 se puede observar que la precisión de las coordenadas es prácticamente 2 veces superior que la incertidumbre de la tendencia.

La solución final obtenida tiene asociado una precisión procedente de la matriz de varianza-covarianza del ajuste final de la solución diaria en el procesado de los datos. Estas precisiones son muy optimistas y no reflejan la verdadera dispersión debido a los errores que no se toman en cuenta en los diferentes modelos usados a lo largo del procesado y a los errores producidos por aquellas causas que no se pueden modelar (García, 2016). Con respecto a la incertidumbre de la tendencia, ésta depende básicamente del número de observaciones de la serie. 
La Figura 3 muestra la serie temporal de la estación CUEC con la tendencia o la evolución a largo plazo, donde se indican sus valores y precisiones correspondientes.
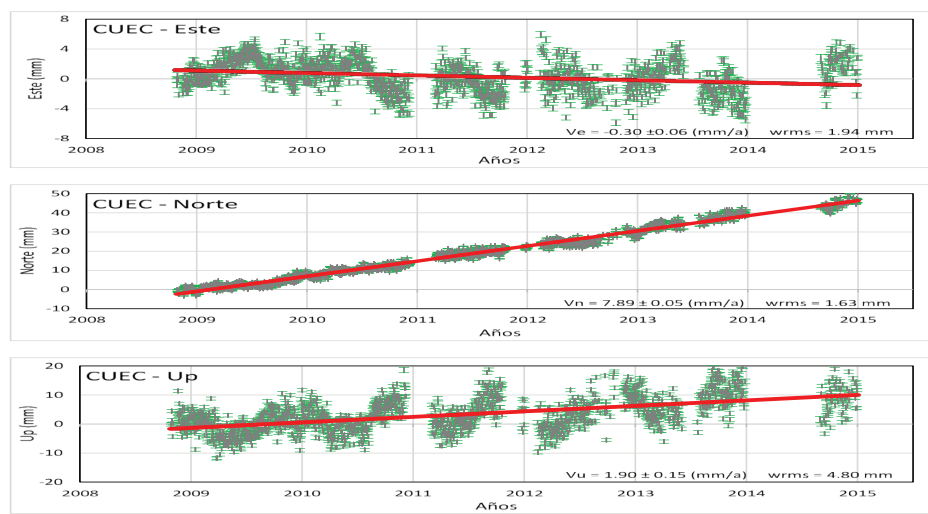

Figura 3. Serie temporal de la estación CUEC en sus tres componentes (e, n, u). Se muestra la tendencia (línea roja) en cada componente, con sus valores e incertidumbres.

\section{ESTACIONALIDAD}

La Figura 4 muestra los periodos predominantes de la estación CUEC donde se puede observar el pico que presenta en el periodo estacional anual ( $\sim 365$ días) en todas sus componentes.

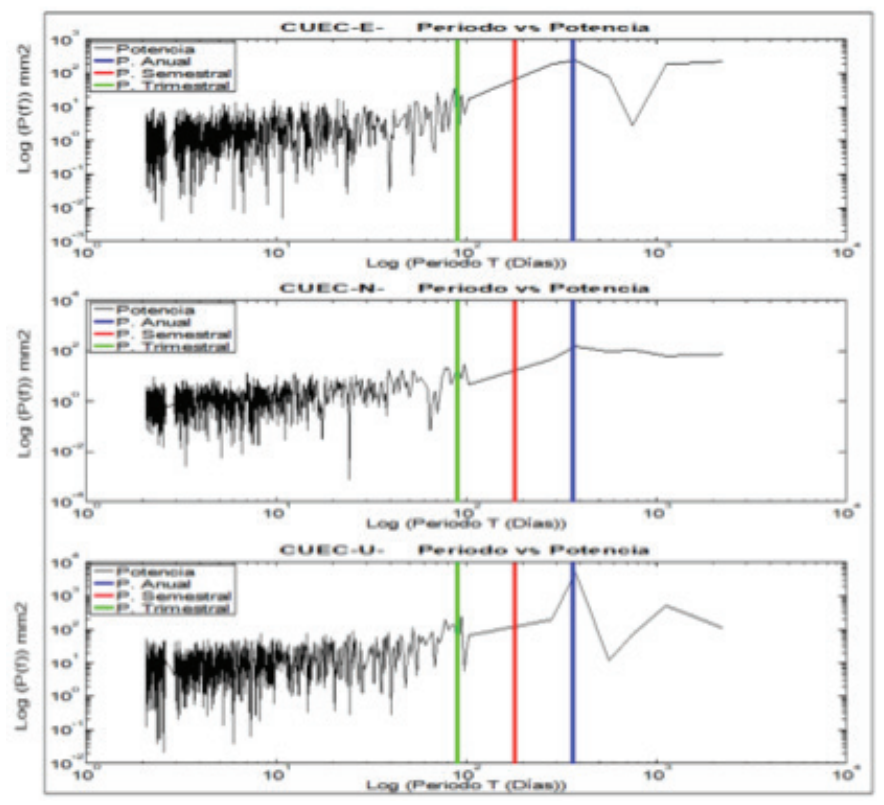

Figura 4. Periodos predominantes de la estación CUEC. Los valores en el eje de las abscisas corresponden al periodo (días) y el eje de las ordenadas al espectro de potencia $\left(\mathrm{mm}^{2}\right)$. Los dos ejes se encuentran en escala logarítmica. 
La Tabla 4 muestra las frecuencias y potencias de observaciones de las series temporales. Para la estación CUEC se obtienen valores de potencia de $245.32 \mathrm{~mm}^{2}, 163.17 \mathrm{~mm}^{2}$ y $4929.50 \mathrm{~mm}^{2}$ para la componente este, norte y vertical, respectivamente. Estos valores son proporcionales a la amplitud de la onda de la serie, es decir a mayor potencia mayor amplitud de onda.

Tabla 4. Frecuencia y potencia de observaciones de la estación CUEC.

\begin{tabular}{ccccccccccc}
\hline & \multicolumn{3}{c}{ Este } & \multicolumn{3}{c}{ Norte } & & Up \\
EST. & $\begin{array}{c}\text { Frec. }(1 / \\
\text { día })\end{array}$ & $\begin{array}{c}\mathrm{P}(\mathrm{f}) \\
(\mathrm{mm} 2)\end{array}$ & $\begin{array}{c}\text { Amp. } \\
(\mathrm{mm})\end{array}$ & $\begin{array}{c}\text { Frec. } \\
(1 / \text { día })\end{array}$ & $\begin{array}{c}\mathrm{P}(\mathrm{f}) \\
(\mathrm{mm} 2)\end{array}$ & $\begin{array}{c}\text { Amp. } \\
(\mathrm{mm})\end{array}$ & $\begin{array}{c}\text { Frec. } \\
(1 / \text { día })\end{array}$ & $\begin{array}{c}\mathrm{P}(\mathrm{f}) \\
(\mathrm{mm} 2)\end{array}$ & $\begin{array}{c}\text { Amp. } \\
(\mathrm{mm})\end{array}$ \\
CUEC & 0.0027 & 245.32 & 0.81 & 0.0027 & 163.17 & 0.66 & 0.0027 & 4929.5 & 3.66 \\
\hline
\end{tabular}

La Figura 5 representa de forma gráfica la estacionalidad en la estación CUEC donde se observa claramente la mayor amplitud de la componente vertical con respecto a las componentes horizontales y la estacionalidad anual como periodo predominante.
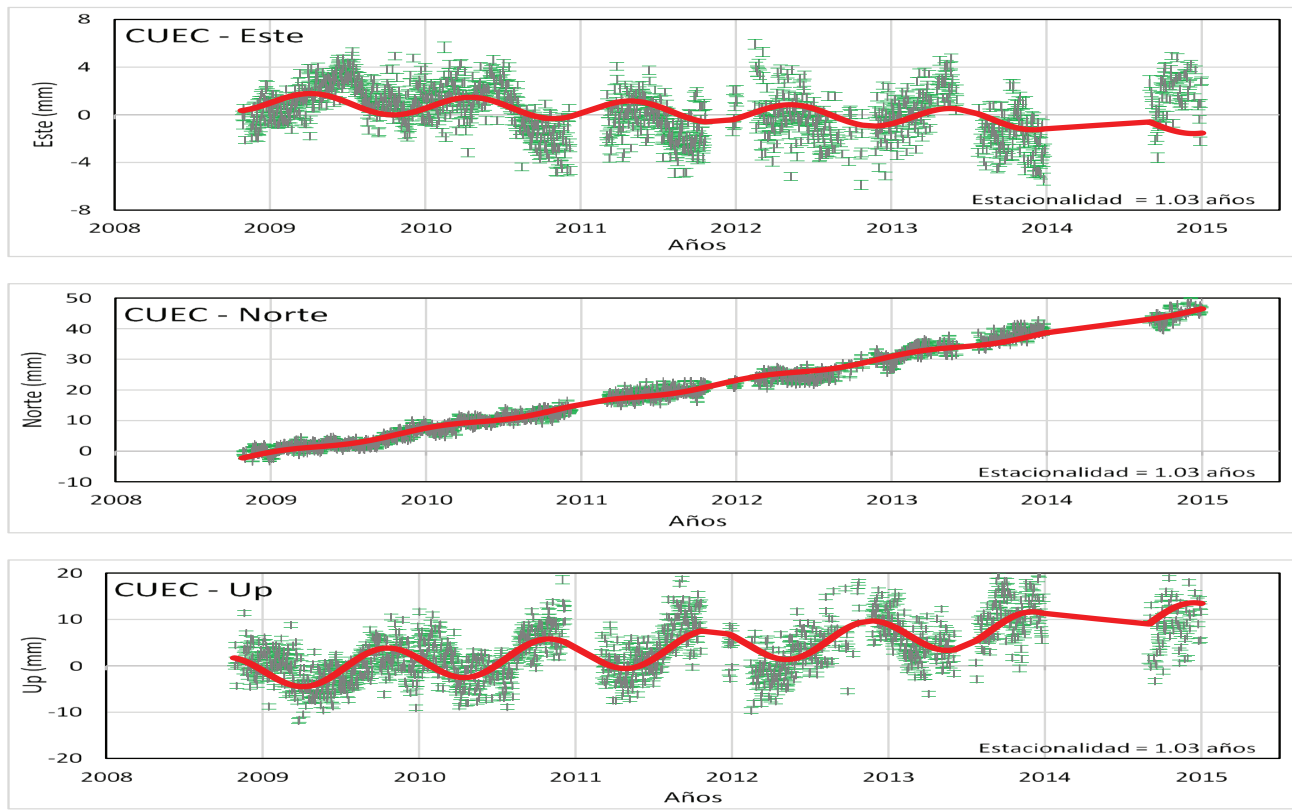

Figura 5. Serie temporal de la estación CUEC en sus tres componentes $(e, n, u)$. Se muestra la estacionalidad (línea roja) en cada componente, con sus valores.

La Tabla 5 muestra los periodos predominantes de la estación CUEC. Se puede observar que los periodos estacionales anuales están presentes en todas sus componenetes. Estudios realizados por Amiri-Simkooei et al. (2007) en series temporales de 10 años, con registros diarios de un conjunto de 71 estaciones con distribución global concluyeron que, para cualquier modelo de ruido, los componentes horizontales tienen menor amplitud de ruido que el componente vertical en el orden de un factor entre 2 y 4. 
Tabla 5. Periodos fundamentales y tiempos de observación de la estación CUEC.

\begin{tabular}{|c|c|c|c|c|c|c|c|}
\hline \multirow{2}{*}{$\begin{array}{l}\text { ESTA- } \\
\text { CIÓN }\end{array}$} & \multicolumn{2}{|c|}{ Este } & \multicolumn{2}{|c|}{ Norte } & \multicolumn{2}{|c|}{ Vertical } & $\begin{array}{c}\text { Tiempo de } \\
\text { observa- }\end{array}$ \\
\hline & $\begin{array}{c}\text { 1er } \\
\text { Periodo } \\
\text { Días }\end{array}$ & $\begin{array}{c}1 \text { er } \\
\text { Periodo } \\
\text { Años } \\
\end{array}$ & $\begin{array}{c}\text { 1er } \\
\text { Periodo } \\
\text { Días } \\
\end{array}$ & $\begin{array}{c}\text { ler } \\
\text { Periodo } \\
\text { Años } \\
\end{array}$ & $\begin{array}{c}\text { 1er } \\
\text { Periodo } \\
\text { Días } \\
\end{array}$ & $\begin{array}{l}\text { 1er Pe- } \\
\text { riodo } \\
\text { Años } \\
\end{array}$ & Años \\
\hline CUEC & 376.00 & 1.03 & 376.00 & 1.03 & 376.00 & 1.03 & 6.18 \\
\hline
\end{tabular}

Amiri-Simkooei et al. (2007) clasifican las estaciones en tres categorías de acuerdo a su periodicidad:

Movimiento periódico del suelo no modelado: En esta categoría se encuentran las series con estacionalidad anual y semianual, se refiere a que el sitio se está moviendo periódicamente (Amiri-Simkooei et al., 2007). Las señales anuales y semianuales, por ejemplo, pueden ser especificadas en esta categoría.

Variación periódica de las series temporales estimadas: Corresponden a estaciones que aparentemente se mueven en forma periódica. Los errores sistemáticos periódicos no modulados (por ejemplo, las mareas) presentes en una estación producirán efectos sistemáticos periódicos más espurios en las series temporales resultantes (Stewart et al., 2005; Amiri-Simkooei et al., 2007).

Las variaciones periódicas que se encuentran correlacionadas con los años de observación: En esta categoría se encuentran las estaciones que tiene como primer periodo fundamental a la mitad del tiempo de observación o al tiempo total de observación.

\section{RUIDO}

El método utilizado para obtener los diferentes tipos de ruido ha sido el análisis espectral para analizar los datos en el dominio de la frecuencia. Se ha utilizado la técnica propuesta por Lomb (1976) para obtener el espectro de potencia en series que no están igualmente espaciadas y luego ajustar a una recta el logaritmo del espectro, donde el valor del índice espectral corresponde a la pendiente de la recta.

Los valores del índice espectral obtenidos para la estación CUEC para sus tres componentes se encontraron en el rango (Tabla 6) que corresponden al ruido gaussiano fraccionario, considerado estacionario, es decir se trata de ruido sin dependencia con el tiempo.

Tabla 6. Valores de índice espectral de la estación CUEC.

\begin{tabular}{cccccccc}
\hline STA- & \multicolumn{2}{c}{ Este, $\boldsymbol{\alpha}$} & \multicolumn{2}{c}{ Norte, $\boldsymbol{\alpha}$} & Up, $\boldsymbol{\alpha}$ \\
TION & \multicolumn{2}{c}{. } & & & & \\
CUEC & -0.46 & \pm & 0.09 & -0.55 & \pm 0.09 & -0.45 & \pm 0.09 \\
\hline
\end{tabular}


La Figura 6 muestra los valores de los índices espectrales para la estación CUEC en cada una de sus componentes, sus valores corresponden a ruido blanco fraccionario.

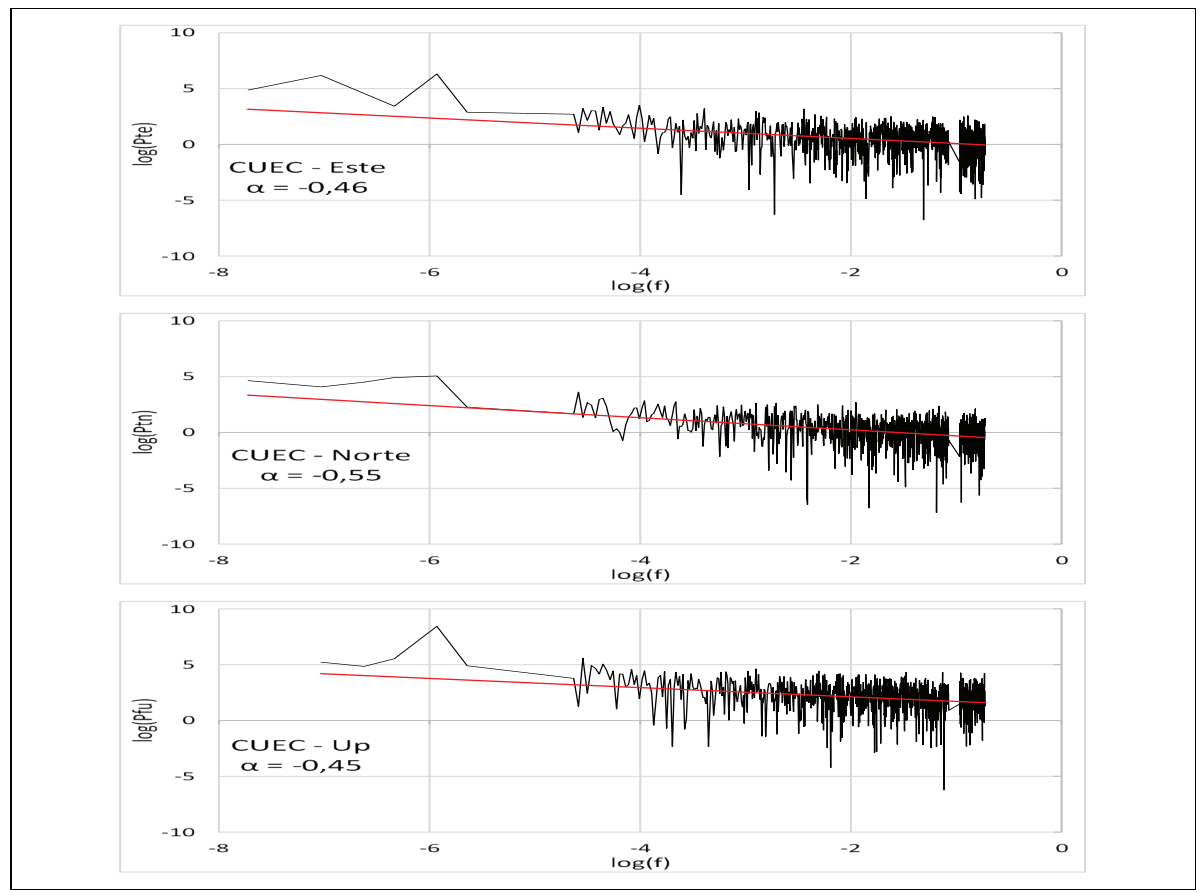

Figura 6. Valores de los índices espectrales para la estación CUEC en cada una de sus componentes, sus valores corresponden a ruido blanco fraccionario. Los valores de ambos ejes se encuentran en escala logarítmica. El eje de las abscisas corresponde al logaritmo de las frecuencias $\left(\mathrm{dia}^{-1}\right)$ y el eje de las ordenadas al logaritmo del espectro de potencia $\left(\mathrm{mm}^{2}\right)$.

Varios autores sugieren que el ruido blanco y ruido rosa dominan el espectro de ruido de coordenadas de estaciones de monitoreo continuo para las series temporales y en una pequeña cantidad el ruido browniano (Zhang et al., 1997; Mao et al., 1999; Williams, 2003b; Williams et al., 2004). Sin embargo, nuestros resultados arrojan ruido gaussiano fraccionario (índice espectral entre 0 y -1) en todas las componentes. Se esperaba encontrar al menos ruido rosa, puesto que la serie tiene periodos de observación de 6 años. La serie de tiempo de las estaciones de monitoreo continuo tienen un comportamiento no lineal complejo y, por lo tanto, el modelo estadístico de la serie de tiempo es más complicado que el simple ruido blanco. Por tanto, suponemos que el valor de ruido obtenido no refleja el verdadero tipo de ruido existente, debido principalmente a los gaps encontrados en cada serie. La estación CUEC inicia sus operaciones en los años 2008 pero tienen un $34.69 \%$ de pérdida de datos. Los índices espectrales determinados por Zhang et al. (1997) a partir del análisis de 10 estaciones con mediciones diarias en 1.6 años de observación varían entre -0.05 y -0.52 con un promedio de -0.40 . Se ha demostrado que para tiempos de observación cortos el ruido blanco es la contribución de ruido dominante, mientras que para las observaciones más largas es el ruido browniano (Hackl et al., 2011). 


\section{CONCLUSIONES}

Se han aplicado dos métodos de eliminación de valores atípicos: primero mediante la aplicación de los procesos integrados que detectan los valores atípicos de dos observaciones consecutivas, algunos de estos valores generalmente no son visibles en la serie porque se encuentran dentro de la tendencia de la misma y luego considerando la tendencia de la serie y eliminando todos aquellos que se encuentren fuera del rango establecido, generalmente tres desviaciones estándar. Para nuestra estación en estudio se detectó un porcentaje del 5.8\% de eliminación de datos, considerado alto si se compara con Nikolaidis (2002), quien alcanzó el nivel promedio de depuración de datos de 1 a $4 \%$.

La detección y corrección de los offsets, así como la eliminación de los valores atípicos encontrados en las series influyen de manera importante en la determinación de los parámetros en estudio, en nuestro caso la estacionalidad, tendencia y tipo de ruido.

La estación CUEC es una estación de monitoreo continuo perteneciente a la categoría de movimiento periódico no modelado de acuerdo a la clasificación de Amiri-Simkooei et al., 2007, por tener una estacionalidad anual.

De acuerdo a varios autores descritos anteriormente el ruido predominante para estaciones de monitoreo continuo es ruido rosa. Sin embargo, nuestros resultados arrojan ruido gaussiano fraccionario (índice espectral entre 0 y -1), se esperaba encontrar al menos ruido rosa, puesto que las series de la estación CUEC tiene un periodo de observación de aproximadamente 6 años. Las series de tiempo de las estaciones de monitoreo continuo tienen un comportamiento no lineal complejo y, por lo tanto, el modelo estadístico de la serie de tiempo es más complejo que el simple ruido blanco. Por ende, suponemos que el valor de ruido obtenido no refleja el verdadero tipo de ruido existente, debido principalmente a los gaps encontrados en la serie (34.69\%). Esta es una desventaja importante en nuestro estudio, lo que significa que la longitud total de la serie no es lo suficientemente larga o está con demasiados valores perdidos dentro de ella que no nos permiten detectar algunos modelos de ruido específicos sobre otros ruidos. Como resultado los parámetros en estudio como la tendencia, estacionalidad, tipo de ruido y velocidades finales pueden estar sesgados.

\section{REFERENCIAS}

Agnew, D. C. (1992). The time-domain behavior of power-law noises. Geophysical research letters, 19(4), 333-336.

Amiri Simkooei, A. R., Tiberius, C. C. J. M., \& Teunissen, S. P. (2007). Assessment of noise in GPS coordinate time series: methodology and results. Journal of Geophysical Research: Solid Earth, 112(B7). DOI: 10.1029/2006JB004913.

Blewitt, G., \& Lavallée, D. (2002). Effect of annual signals on geodetic velocity. Journal of 
Geophysical Research: Solid Earth, 107(B7). DOI: 10.1029/2001JB000570.

Bos, M. S., Fernandes, R. M. S., Williams, S. D. P., \& Bastos, L. (2008). Fast error analysis of continuous GPS observations. Journal of Geodesy, 82(3), 157-166. DOI: 10.1007/s00190007-0165-x.

García, L., (2016) Análisis de series temporales en estaciones permanentes GPS. Tesis Doctoral. Universidad Complutense de Madrid. Facultad de Ciencias Matemáticas. Madrid, España.

Drewes, H., O. (2014). Curso Sistemas de Referencia. Deutsches Geodätisches Forschungsinstitut - DGFI, International Association of Geodesy - IAG, Sistema de Referencia Geocéntrico para las Américas - SIRGAS.

Hackl, M., Malservisi, R., Hugentobler, U., \& Wonnacott, R. (2011). Estimation of velocity uncertainties from GPS time series: Examples from the analysis of the South African TrigNet network. Journal of Geophysical Research: Solid Earth, 116(B11). DOI: $10.1029 / 2010 J B 008142$.

Johnson, H. O., \& Agnew, D. C. (1995). Monument motion and measurements of crustal velocities. Geophysical Research Letters, 22(21), 2905-2908.

Lomb, N. R. (1976). Least-squares frequency analysis of unequally spaced data. Astrophysics and space science, 39(2), 447-462.

Mao, A., Harrison, C. G., \& Dixon, T. H. (1999). Noise in GPS coordinate time series. Journal of Geophysical Research: Solid Earth, 104(B2), 2797-2816.

Nikolaidis, R (2002). Observation of Geodetic and Seismic Deformation with the Global Positioning System. Ph.D. thesis, University of California.

Peña, D. (2010). Análisis de series temporales. Madrid - España. Alianza Editorial S.A.

Press, W. H., Teukolsky, S. A., Vetterling, W. T., \& Flannery, B. P. (2007). Numerical recipes third edition: the art of scientific computing. Cambridge University Press, 32, 10013-2473.

Scargle, J. D. (1982). Studies in astronomical time series analysis. II-Statistical aspects of spectral analysis of unevenly spaced data. The Astrophysical Journal, 263, 835-853.

Stewart, M. P., N. T. Penna, and D. D. Lichti (2005), Investigating the propagation mechanism of unmodelled systematic errors on coordinate time series estimated using least squares, $\mathrm{J}$. Geod., 79, 479 - 489, doi:10.1007/s00190-005-0478-6.

Williams, S. D. (2003b). Offsets in global positioning system time series. Journal of Geophysical Research: Solid Earth, 108(B6).

Williams, S. D., Bock, Y., Fang, P., Jamason, P., Nikolaidis, R. M., Prawirodirdjo, L., Miller, M. \& Johnson, D. J. (2004). Error analysis of continuous GPS position time series. Journal of Geophysical Research: Solid Earth, 109(B3).

Wolf, P. R., \& Ghilani, C. D. (1997). Adjustment computations: statistics and least squares in surveying and GIS. Wiley-Interscience.

Zhang, J., Bock, Y., Johnson, H., Fang, P., Williams, S., Genrich, J.,.. \& Behr, J. (1997). Southern California Permanent GPS Geodetic Array: Error analysis of daily position estimates and site velocities. Journal of Geophysical Research: Solid Earth, 102(B8), 18035-18055. DOI: $10.1029 / 97 J B 01380$. 